\title{
Proyecto Pedagógico: una visión en prospectiva hacia el mejoramiento continuo*
}

\author{
Educational Project: A prospective vision towards \\ continuous improvement
}

\section{Projeto Educacional: Uma visão prospectiva para a melhoria contínua}

\author{
Liliana María Cardona Mejía** \\ Universidad de Antioquia, Medellín, Colombia
}

Resumen. Las prácticas pedagógicas han sido objeto de indagación de muchos autores; sin embargo, en la búsqueda realizada, fueron pocos los estudios hallados referidos al ejercicio que desarrollan los estudiantes de los últimos niveles de formación universitaria, pues la mayoría se refieren al quehacer docente. Barragán (2012), por ejem-

* Artículo de Investigación. Este artículo se deriva de la investigación Las Prácticas Pedagógicas en Educación Física: Un Análisis a la luz de la Licenciatura en Educación Física y Deportes de la Universidad de San Buenaventura- Seccional Medellín, desarrollada durante los años 2010-2011 y adscrita al grupo de investigación Estudios Interdisciplinarios Sobre Educación- ESINED de la Facultad de Educación de la Universidad de San Buenaventura, Medellín, en el marco del macroproyecto: La gestión educativa y el currículo en las prácticas contemporáneas, de la línea Gestión Educativa y Currículo.

** Licenciada en Educación Física y Especialista en Administración Deportiva de la Universidad de Antioquia. Magister en Educación de la Universidad de San Buenaventura. Profesora Instituto Universitario de Educación Física de la Universidad de Antioquia. Coordinadora Grupo de Investigación en Ciencias aplicadas a la Actividad Física y el Deporte GRICAFDE. Asesora y consultora de proyectos Universidad de San Buenaventura. E-mail: lili.cardona25@gmail.com 
plo, es uno de los autores que reconoce que el tema de la práctica pedagógica ha sido bastante controvertido y trasciende el concepto de lo práctico, de la técnica, hacia un aspecto de disposición humana en relación con el conocimiento y en búsqueda de un bien que involucra lo moral, lo ético y lo político. Desde un contexto general su puede mencionar un estudio que realizó la Universidad Pedagógica Nacional, logrando una caracterización de las prácticas en todos los programas de pregrado, de donde se destacan hallazgos como la diversidad de términos para referirse al tema, algunas categorías similares a las del objeto del presente estudio y los escenarios de práctica dejando una duda de la manera cómo se determina la idoneidad o no del centro. Gaitán et al (2005) realizó un trabajo de categorización de la práctica de donde se desprenden términos como práctica educativa, práctica docente, prácticas de enseñanza, entre otros. Este trabajo confirma la diversidad de términos para referirse al tema, con elementos comunes en todos ellos; sin embargo, en la investigación se determinó utilizar el término práctica pedagógica entendida como todas aquellas acciones que se desarrollan en un espacio educativo, llámese escuela, institución, empresa o cualquier tipo de organización donde se generen procesos formativos. El estudio pretendió caracterizar el proceso de prácticas que se desarrolla en el pregrado de Educación Física y Deportes de la Universidad de San Buenaventura de Medellín en aras de generar una propuesta alternativa desde una perspectiva contemporánea del currículo. En un primer momento se presentan los resultados relacionados con la estructura académico - administrativa de la práctica; luego se aborda el tema de los escenarios y las percepciones de directivos, docentes y estudiantes sobre el proceso y finalmente, se presente una propuesta de nuevas formas de pensar la práctica en perspectiva del mejoramiento continuo.

Palabras clave. Práctica pedagógica, educación física, currículo (Tesauro Unesco).

Abstract. Pedagogical practices have been the subject of investigation of many authors, but the search was performed, few studies were found relating to the year that students develop training recent college levels, as most refer to teaching work. Barragán (2012), for example, is an author who recognizes that the issue of pedagogical practice has been quite controversial and transcends the concept of practicality, technology, to an aspect of human disposition in relation 
to knowledge and in search of a good that involves moral, ethical and political. From a general context can mention the study by the National Pedagogical University, obtaining a characterization of the practice in all undergraduate programs of findings highlighting the diversity of terms to refer to the subject, some categories similar to those of purpose of the present study and practice settings leaving a doubt the way how to determine the suitability or otherwise of the center. Gaitán et al (2005) conducted a categorization work practice which emerges terms as educational practice, teaching practice, teaching practices, among others. This work confirms the variety of terms to refer to the subject, with common elements in all of them, however, the investigation was determined to use the term teaching practice understood as all actions that take place in an educational space, be it school, institution, company or any organization that generates training processes. The study sought to characterize the process of practice that develops in the undergraduate Physical Education and Sports, University of San Buenaventura de Medellín in order to generate an alternative proposal from a contemporary perspective of the curriculum. At first we present the results related to the academic structure - practice management and then addresses the issue of the scenarios and the perceptions of principals, teachers and students about the process and finally present a proposal for new forms of thinking practice continuous improvement perspective.

Keywords. Pedagogical practice, physical education, curriculum. curriculum (Thesaurus Unesco).

Resumo. Práticas pedagógicas têm sido objeto de investigação de muitos autores, mas a pesquisa foi realizada, poucos estudos foram encontrados sobre o ano em que os alunos desenvolvem níveis recentes da faculdade de treinamento , como a maioria referem-se a trabalho docente. Barragán (2012), por exemplo, é um autor que reconhece que a questão da prática pedagógica tem sido bastante polêmico e transcende o conceito de praticidade, tecnologia, para um aspecto da disposição humana em relação ao conhecimento e em busca de um bem que envolve moral , ético e político. A partir de um contexto geral, pode-se mencionar é um estudo da Universidade Pedagógica Nacional, as obtenções de uma caracterização da prática em todos os cursos de graduação das descobertas destacam a diversidade de termos para se referir ao assunto, algumas categorias semelhantes às de objetivo 
do presente estudo e na prática as definições deixando uma dúvida a maneira como determinar a adequação ou não do centro. Gaitán et al (2005) realizaram uma prática de trabalho categorização que surgem termos como prática educativa, as práticas pedagógicas, práticas de ensino, entre outros. Este trabalho confirma a variedade de termos para se referir ao assunto, com elementos comuns em todos eles, no entanto, a investigação foi determinada a usar a prática docente termo entendido como todas as ações que ocorrem em um espaço educativo, seja escola, instituição, empresa ou qualquer organização que gera processos de formação. O estudo procurou caracterizar o processo de prática que se desenvolve na graduação de Educação Física e Esporte da Universidade de San Buenaventura de Medellín, a fim de gerar uma proposta alternativa a partir de uma perspectiva contemporânea do currículo. Na primeira são apresentados os resultados relativos à estrutura acadêmica - práticas de gestão e, em seguida, aborda a questão dos cenários e as percepções dos diretores, professores e alunos sobre o processo e, finalmente, apresentar uma proposta de novas formas de pensando perspectiva de melhoria contínua prática.

Palavras chave. Prática de ensino, currículo de educação física (Tesauro Unesco).

\section{Introducción}

La investigación Las prácticas pedagógicas en Educación Física: Un análisis a la luz de la Licenciatura en Educación Física y Deportes de la Universidad de San Buenaventura- Seccional Medellín pretendió analizar la estructura académico - administrativa de la práctica pedagógica, identificar los escenarios de práctica, describir las percepciones que tienen directivos, profesores y estudiantes sobre la práctica pedagógica y generar una propuesta académica y administrativa para la práctica pedagógica en la Licenciatura en Educación Física y Deportes de la Universidad de San Buenaventura, Seccional Medellín.

El enfoque del estudio fue de tipo cualitativo, específicamente estudio de caso, con una población conformada por directivos, docentes y estudiantes de la licenciatura en educación física y deportes de la Universidad de San Buenaventura, Seccional Medellín y utilizando como técnicas principales la entrevista y la revisión documental; como complemento al proceso y reconociendo lo cuantitativo como dialógico 
más que contradictorio, se aplicaron también algunas encuestas a los estudiantes.

Las técnicas que se utilizaron para recoger la información fueron: entrevista a directivos, entrevista a grupo focal de docentes, encuesta a estudiantes y análisis documental.

\section{Desarrollo}

En el presente artículo se hace énfasis en los resultados de la investigación; sin embargo, para ubicar en términos conceptuales al lector, se hace un breve recorrido por algunos de los fundamentos teóricos que soportaron el proceso como son práctica pedagógica y currículo.

Se reconoce la multiplicidad de términos para referirse a la práctica desde una perspectiva pedagógica tales como práctica educativa, práctica docente, prácticas de enseñanza y práctica pedagógica en sí misma; sin embargo, no se encuentran elementos significativamente diferenciadores. En este sentido, se retoma el trabajo de Gaitán et al (2005) que relaciona la práctica con conocimiento y con cultura, en tanto hay una manera heredada de hacer las cosas. Se destacan los aportes de Vain, citado por Gaitán et al (2005), que menciona cuatro dimensiones de la práctica, como son los actores (alumnos, docentes, conocimiento), los escenarios (instituciones educativas, sociedad), la trama (grupos y relaciones) y de manera significativa para la línea de investigación, el currículo. Se relaciona el concepto de práctica pedagógica o prácticas docentes con el de currículo entendiéndolo no solo como plan de estudios, sino como proceso que se construye a la par que se recorre, es decir, desde una perspectiva práctica donde se desarrollan acciones, eventos, sucesos en el contexto donde se instaura el proceso de enseñanza - aprendizaje.

Son diversas las denominaciones que se encuentran sobre currículo, entre las que se destacan las de Moreno y Pulido (2009), que expresan que el currículo debe ser entendido como la selección de cultura que se hace para la formación de otro o con otro; la de Sacristán (2010) que menciona que el currículo se constituye en la herramienta de regulación de las prácticas pedagógicas y que hoy debe ir más allá de los contenidos y la de Álvarez y González (2002) que reconocen que en un primer momento el currículo era concebido solo como el plan 
de estudios cuyo fin era la formación de ciudadanos atendiendo las necesidades del Estado y de la Iglesia, pero luego por influencias de corrientes como la pedagogía y la psicología, el currículo fue concibiéndose como un conjunto de experiencias, hechos, acontecimientos, saberes y destrezas que trascienden el solo plan de estudios; definición que se acogió en la investigación.

Se presentan ahora, los principales hallazgos que se desprendieron de algunas categorías, como son la estructura académico administrativa, los escenarios y las percepciones sobre la práctica.

\section{Estructura académico - administrativa}

La Licenciatura en Educación Física y Deportes de la Universidad de San Buenaventura es uno de los cinco programas adscritos a la facultad de Educación; la práctica pedagógica, en este sentido, se acoge al esquema de facultad en donde hay una coordinadora de prácticas para las cinco licenciaturas que atiende a más de 500 estudiantes. La denominación que se le da al proceso de prácticas en la facultad, es la de proyecto pedagógico; no obstante, la denominación que hace la Universidad según el reglamento de práctica, es la de prácticas profesionalizantes.

La práctica tiene presencia en los diez niveles del plan de estudios, siendo este un elemento diferenciador de la facultad de educación, dado que en las otras facultades solo se hacen prácticas profesionalizantes en los últimos semestres.

Se identifican tres momentos en la práctica que son el de fundamentación, profundización y especialización y cuatro modalidades, como son docente, gestión pedagógica, social comunitaria e investigativa.

La distribución en el plan de estudios es la siguiente:

Prácticas pedagógicas formativas y de profundización:

- Epistemología y cognición

- Investigación Educativa

- Investigación pedagógica 
- Proyecto pedagógico y Escenarios

- Proyecto pedagógico y Rol del Maestro

- Proyecto pedagógico y saberes específicos

Prácticas pedagógicas profesionalizantes:

- Proyecto Pedagógico I

- Proyecto pedagógico II

- Proyecto pedagógico III

- Proyecto pedagógico IV

- Trabajo de grado I

- Trabajo de grado II

Los estudiantes seleccionan libremente la modalidad en la que quieren incursionar sin un orden lineal, lo que no implica necesariamente rotación por todas las modalidades, dado que pueden estar en la misma modalidad semestre tras semestre; sin embargo, atendiendo a una política institucional, deben estar un año continuo en la modalidad docente.

La Licenciatura en Educación Física y Deportes debe ajustar sus formas de práctica específica desde las áreas de desempeño que la componen (escolar, actividad física y salud, entrenamiento deportivo, recreación y administración deportiva), lo que en ocasiones cuesta un poco como es el caso por ejemplo, de entrenamiento deportivo, en tanto tiene unas características específicas que no se articulan completamente con ninguna de las modalidades establecidas; sin embargo, la práctica en entrenamiento deportivo se considera dentro de la modalidad docente.

Algo similar ocurre con el área de actividad física y salud, dado que se considera una práctica en lo social comunitario; sin embargo, el enfoque de esta modalidad es población vulnerable y hay prácticas que se desarrollan con otro tipo de población para el mantenimiento de la salud o el buen aprovechamiento del tiempo libre.

El área de la gestión deportiva se incluye en la modalidad de gestión escolar limitando escenarios como clubes deportivos, entes deportivos, ligas, entre otros.

Los actores que intervienen en el proceso son: una coordinadora de prácticas, un asesor, un representante del centro de práctica, el estudiante y 
los usuarios. Se menciona el director de programa como actor principal; sin embargo solo interviene de ser necesario, para atender dificultades que se presenten en el proceso.

En cuanto al procedimiento para solicitar un centro de práctica, cada estudiante debe contactar a la coordinadora de manera personal o telefónica, para solicitarlo de acuerdo con un listado de centros que se publica en un blog de la práctica; luego, si hay disponibilidad, la coordinadora envía hoja de vida y el estudiante se somete a un proceso de selección.

El acompañamiento que hace el asesor al estudiante es grupal para los niveles 1 a 6 y puede tener una asignación de hasta 20 estudiantes según la coordinación de prácticas; sin embargo, la población entrevistada de docentes menciona grupos hasta de 35 estudiantes.

En un mismo grupo pueden haber diversas modalidades de práctica; por lo tanto, se espera que el docente al frente tenga dominio de todas las modalidades, lo cual, por las diversas áreas y campos de acción de la educación física, es poco probable.

Otra de las políticas institucionales es que el asesor de práctica, bajo el argumento de la autonomía de los estudiantes y de no convertirse en un supervisor, hace solo una visita inicial y otra final al centro; por lo tanto los procesos de realimentación se dan a partir de conversaciones con el cooperador -que por lo general no es del área- y con el estudiante.

La evaluación del proceso tiene varios componentes: el seguimiento que hace el asesor, la evaluación que el representante del centro hace al estudiante y la presentación de un proyecto pedagógico que debe ejecutarse. El instrumento para la evaluación del asesor y del representante es un cuestionario que es genérico para todos los programas de la facultad; en el caso del proyecto pedagógico, no existe un formato de presentación establecido y se confunde con un formato de informe final que deben presentar los estudiantes.

\section{Escenarios de práctica}

Los escenarios de práctica son los lugares donde los estudiantes realizan las prácticas tales como instituciones educativas de todos los niveles de 
escolaridad, institutos municipales de deporte y recreación, hospitales, corporaciones, universidades y organizaciones o empresas.

No se encontraron estudios de caracterización de los escenarios; la información de los centros se reduce a un listado que se publica en el blog de prácticas.

En su orden, los escenarios que más reconocen los estudiantes para desarrollar sus prácticas pedagógicas, son las instituciones educativas públicas, los gimnasios y las ligas deportivas, y los de más bajo reconocimiento son los grupos de investigación.

En cuanto al requisito para ser centro de práctica se muestran varias formas; una es que el centro de práctica haga la solicitud y la universidad hace una visita para analizar sus necesidades y posibles modalidades; otra es que el centro de práctica es propuesto por el docente quien labora allí y en este caso se prescinde de la visita institucional al centro confiando en el criterio del docente. En ocasiones, de acuerdo con las necesidades específicas de formación de los estudiantes, la universidad busca los centros y otra forma es que los estudiantes también pueden proponer un centro.

Estas formas fueron descritas por los sujetos investigados pero no se encontró una reglamentación específica que describa qué debe cumplir un centro para ser considerado centro de práctica.

\section{Percepciones}

Desde la perspectiva de los directivos y docentes se cruzan varias percepciones, que se considera pertinente enumerar:

- $\quad$ El proceso presenta debilidades en claridad tanto para el estudiante como para el docente que llega.

- Las responsabilidades administrativas son inmensas para ser asumidas por una sola persona.

- $\quad$ Se requiere una mayor claridad para el docente en cuanto a los contenidos y actividades a desarrollar.

- La asignación del centro de práctica se hace en muchas ocasiones de manera tardía, citando casos de estudiantes sin centro transcurrido un mes o hasta dos meses de iniciado el semestre. 
- Se habla de niveles de proyecto pedagógico pero semestre tras semestre se ve al estudiante haciendo lo mismo, planteando lo mismo.

- No hay un orden o una estructura en el proceso que permita ir haciendo prácticas de manera coherente con el plan de estudios.

- Por la normatividad de tener que estar un año en docencia y la no estructuración secuencial del proceso, se ven estudiantes de nivel 9 apenas pensando en este tipo de práctica para poder cumplir con el requisito.

- Es muy difícil que un solo docente haga seguimiento a diferentes modalidades de práctica en el mismo grupo, lo que genera dudas en el acompañamiento cuando toca asesorar áreas que no son del dominio del docente.

- A veces la labor del docente termina siendo una asesoría a la construcción del proyecto pedagógico y no de la práctica directa del estudiante.

- Los formatos pudieran dinamizar el proceso pero es difícil para una sola coordinación lograr su sistematización y que no se queden allí.

En el caso de los estudiantes el $48 \%$ de ellos manifiesta estar muy de acuerdo o de acuerdo con que el proceso de prácticas es adecuado, el $21 \%$ está en desacuerdo o muy en desacuerdo y el $12 \%$ no está ni de acuerdo ni en desacuerdo.

En relación con el acompañamiento que reciben de la coordinación de prácticas, los estudiantes los valoran como suficiente en un $35 \%$ y en un porcentaje similar (31\%) como insuficiente.

\section{La propuesta}

Como producto del proceso de investigación, se plantea una propuesta que pretende contribuir con el mejoramiento continuo de los procesos de práctica al interior de la licenciatura en Educación Física y Deportes de la Universidad de San Buenaventura - Seccional Medellín, 
optimizando los procesos y aumentando los niveles de satisfacción de estudiantes.

Un primer elemento: la especificidad de la educación física y de sus áreas implica contemplar modalidades propias para las prácticas en la Licenciatura Educación Física, tales como:

- Docencia

- Actividad física y promoción de la salud

- Entrenamiento deportivo

- Recreación

- Gestión deportiva

- Investigación

Así mismo, para ser coherentes con el plan de formación y para mayor control y acompañamiento del proceso, se recomienda establecer un orden en las modalidades de práctica y no dejarlo a la voluntad del estudiante, iniciando con la modalidad docente como base del proceso pedagógico, siendo un programa adscrito a una facultad de educación. Además, esto facilitaría la asignación de los profesores con perfiles específicos para la modalidad que se desarrolle por nivel; sin embargo, el alto número de estudiantes en un grupo atendidos por un solo docente, sigue siendo un asunto problemático que podría intervenirse con varios profesores.

Ayudaría a agilizar el procedimiento de inscripción a la práctica y elección del centro, el contar con un sistema de información que permita tener la información disponible y actualizada.

De otro lado, se debe tener en cuenta que la Educación Física tiene un componente procedimental, es decir, práctico, bastante alto, lo que genera unas condiciones de intervención diferentes en relación con las demás licenciaturas; una muestra de ello es el escenario en sí mismo, pues deja de ser el aula y se privilegian los escenarios deportivos. En este sentido, se hace necesaria una mayor presencia del docente en el centro, no para supervisar sino para acompañar y orientar la práctica del estudiante.

De igual manera, se hace necesaria una caracterización de los escenarios de práctica que permita comprender a profundidad las características 
con que cuentan y las opciones y posibilidades de desarrollo que le ofrecerían a los estudiantes.

Se requiere hacer un trabajo de sensibilización en los primeros niveles de formación y con los docentes nuevos, para entender la significación y dinámica de proyecto pedagógico y de esta manera, unificar también criterios de orientación de proyecto pedagógico para que no sean solo a juicio del docente.

Una coordinación para todas las prácticas de la facultad es insuficiente, se sugiere el acompañamiento de por lo menos un docente en cada programa, para que desde cada proyecto educativo de programa en su especificidad, aporten dominio conceptual, procedimental, actitudinal y transversal a partir del campo específico de formación.

Desde el aspecto académico, se sugiere hacer ajustes al plan de formación, que permiten una articulación real y coherente entre teoría y práctica; se sugiere la siguiente adición de contenidos:

\section{Cuadro I.}

Propuesta de adición de contenidos al plan de estudios

\begin{tabular}{|c|c|}
\hline Asignatura & $\begin{array}{c}\text { Propuesta de adición de contenidos } \\
\text { (conceptuales, procedimentales } \\
\text { y actitudinales) }\end{array}$ \\
\hline $\begin{array}{l}\text { Epistemología } \\
\text { y cognición }\end{array}$ & $\begin{array}{l}\text { Fundamentación teórica del proceso de proyecto } \\
\text { pedagógico en la Universidad y la facultad. Como: } \\
\text { - Denominaciones } \\
\text { - Reglamentos } \\
\text { - Procesos (investigación, intervención, acadé- } \\
\text { - } \text { micos, administrativos) } \\
\text { - Pribilidad }\end{array}$ \\
\hline
\end{tabular}




\begin{tabular}{|c|c|}
\hline $\begin{array}{l}\text { Investigación } \\
\text { pedagógica }\end{array}$ & $\begin{array}{l}\text { Fundamentación teórica del proceso de proyecto } \\
\text { pedagógico en el programa de licenciatura en edu- } \\
\text { cación física y deportes. Como: } \\
\text { Bases para la investigación pedagógica (ejercicio } \\
\text { de investigación cualitativa) } \\
\text { Diseños de investigación (etnografía, fenomenolo- } \\
\text { gía, hermenéutica, estado del arte, estudio de caso, } \\
\text { investigación acción-participación) } \\
\text { Técnicas e instrumentos de recolección de infor- } \\
\text { mación (observación: diario de campo, guía de } \\
\text { observación, notas de campo, entrevista: guía de } \\
\text { cuestionario individual - grupal) } \\
\text { - } \quad \text { La ética en la investigación } \\
\text { Escenarios de práctica } \\
\text { - Actores de los procesos de proyecto pedagógico } \\
\text { - Denominaciones de las áreas del campo } \\
\text { - Finalidades Educativas de las áreas del campo } \\
\text { - Procesos escriturales: citaciones }\end{array}$ \\
\hline $\begin{array}{l}\text { Investigación } \\
\text { educativa }\end{array}$ & $\begin{array}{l}\text { Fundamentos procedimentales, actitudinales de } \\
\text { intervención y participación en los procesos y acti- } \\
\text { vidades de proyecto pedagógico. Como: } \\
\text { - } \quad \text { Bases para la investigación educativa (ejercicio } \\
\text { de investigación cuantitativa) } \\
\text { - } \quad \text { Diseños de investigación (no experimental: } \\
\text { - } \quad \text { descriptivo y correlativo) } \\
\text { - } \quad \text { Estactest, escalas de valoración } \\
\text { - } \quad \text { Docentes y estudiantes en eventos académicos } \\
\text { - } \quad \text { Perfil de docentes y estudiantes en el programa } \\
\text { - } \quad \text { Finalidades Pedagógicas de las áreas del campo } \\
\text { - } \quad \text { Procesos escriturales: búsqueda de información } \\
\text { en bases de datos }\end{array}$ \\
\hline
\end{tabular}




\begin{tabular}{|c|c|}
\hline $\begin{array}{l}\text { Proyecto } \\
\text { pedagógico y } \\
\text { rol del maestro }\end{array}$ & $\begin{array}{l}\text { Fundamentos teóricos sobre el rol de ser maestro a } \\
\text { través de diferentes épocas, escenarios y contextos } \\
\text { para la educación física, la actividad física, el de- } \\
\text { porte, la recreación, la administración deportiva, la } \\
\text { psicología deportiva y la investigación en el sector. } \\
\text { Como: } \\
\text { - Maestro } \\
\text { - } \quad \text { Entrenador } \\
\text { - } \quad \text { Instructor } \\
\text { - } \quad \text { Administrador } \\
\text { - } \quad \text { Ludotecario - Recreacionista } \\
\text { - } \quad \text { Asistente - preparador físico } \\
\text { - } \quad \text { Asistente - psicología deportiva } \\
\text { - Investigador } \\
\text { - Diseño, aplicación y análisis de instrumentos } \\
\text { - } \quad \text { Procesos escriturales: cómo hacer un referente } \\
\text { teórico-conceptual }\end{array}$ \\
\hline $\begin{array}{l}\text { Proyecto } \\
\text { pedagógico y } \\
\text { escenarios }\end{array}$ & $\begin{array}{l}\text { Fundamentos teóricos y prácticos sobre el rol de ser } \\
\text { licenciado en diferentes escenarios y contextos de la } \\
\text { educación física, la actividad física, el deporte, la re- } \\
\text { creación y de manera transversal, la administración } \\
\text { deportiva, la psicología deportiva y la investigación } \\
\text { en el sector, como: } \\
\text { - } \quad \text { Instituciones Educativas } \\
\text { - } \quad \text { Organizaciones deportivas } \\
\text { - Organizaciones de actividad física y promoción } \\
\text { - de la salud } \\
\text { - } \quad \text { Erganizaciones recreativas } \\
\text { po, línea, proyecto, semillero y visibilidad de } \\
\text { productos) } \\
\text { Diseño, aplicación y análisis de instrumentos } \\
\text { cualitativos y/o cuantitativos y argumentación } \\
\text { Procesos escriturales: principales normas para } \\
\text { trabajos escritos }\end{array}$ \\
\hline
\end{tabular}




\begin{tabular}{|c|c|}
\hline $\begin{array}{l}\text { Proyecto } \\
\text { pedagógico y } \\
\text { saber } \\
\text { específico }\end{array}$ & $\begin{array}{l}\text { Fundamentos conceptuales, procedimentales y ac- } \\
\text { titudinales sobre el perfil del educador físico en la } \\
\text { Recreación escolar en el sector público o privado, } \\
\text { según el nivel de formación. } \\
\text { - } \quad \text { Procesos administrativos de planeación, segui- } \\
\text { miento y evaluación en las Instituciones u orga- } \\
\text { nizaciones centros de práctica de los estudiante } \\
\text { - Reconocimiento de los diferentes roles dentro } \\
\text { del saber específico } \\
\text { Diseño, aplicación, análisis de instrumentos } \\
\text { cualitativos y/o cuantitativos, argumentación y } \\
\text { creación de propuesta de intervención } \\
\text { Ejercicios de intervención acompañados } \\
\text { Procesos escriturales: lista de referentes }\end{array}$ \\
\hline $\begin{array}{l}\text { Proyecto } \\
\text { pedagógico I }\end{array}$ & $\begin{array}{l}\text { Fundamentos conceptuales, procedimentales y } \\
\text { actitudinales sobre el perfil del educador físico en } \\
\text { la educación física escolar en el sector público o } \\
\text { privado, según el nivel de formación. } \\
\text { - Procesos escriturales: aplicación }\end{array}$ \\
\hline $\begin{array}{l}\text { Proyecto } \\
\text { pedagógico II }\end{array}$ & $\begin{array}{l}\text { Fundamentos conceptuales, procedimentales y } \\
\text { actitudinales sobre el perfil del educador físico en } \\
\text { el entrenamiento deportivo en el sector público o } \\
\text { privado, según el nivel de formación. } \\
\text { - Procesos escriturales: aplicación }\end{array}$ \\
\hline $\begin{array}{l}\text { Proyecto } \\
\text { pedagógico III }\end{array}$ & $\begin{array}{l}\text { Fundamentos conceptuales, procedimentales y ac- } \\
\text { titudinales sobre el perfil del educador físico en la } \\
\text { actividad física y la promoción de la salud en el sec- } \\
\text { tor público o privado, según el nivel de formación. } \\
\text { - Procesos escriturales: aplicación }\end{array}$ \\
\hline
\end{tabular}




\begin{tabular}{|l|l|}
\hline \multirow{2}{*}{$\begin{array}{l}\text { Proyecto } \\
\text { pedagógico IV }\end{array}$} & $\begin{array}{l}\text { Fundamentos conceptuales, procedimentales y ac- } \\
\text { titudinales sobre el perfil del educador físico en la } \\
\text { o la investigación a partir de la educación física } \\
\text { escolar, la actividad física, el deporte o la recreación } \\
\text { en el sector público o privado según la elección del } \\
\text { estudiante. }\end{array}$ \\
Procesos escriturales: aplicación
\end{tabular}

Finalmente, se presenta una propuesta de visibilidad para nuevas modalidades y centros de práctica:

\section{Cuadro 2.}

Nuevas modalidades y escenarios de práctica

\begin{tabular}{|c|c|}
\hline $\begin{array}{c}\text { Nuevas } \\
\text { modalidades }\end{array}$ & Nuevos centros de práctica \\
\hline $\begin{array}{l}\text { Entrenamiento } \\
\text { deportivo }\end{array}$ & $\begin{array}{ll}\text { - } & \text { Clubes deportivos } \\
\text { - } & \text { Ligas deportivas } \\
\text { - } & \text { Institutos de deporte } \\
\text { - } & \text { Centros penitenciarios } \\
& \text { centros de inclusión para población en situa- } \\
& \end{array}$ \\
\hline $\begin{array}{l}\text { Actividad física } \\
\text { y salud }\end{array}$ & $\begin{array}{ll}\text { - } & \text { Centros educativos del programa municipal de } \\
\text { - } & \text { Juen comienzo } \\
\text { - } & \text { Unidades residenciales } \\
\text { - } & \text { Centros penitenciarios } \\
\text { - } & \text { Centros de inclusión para población en situa- } \\
\text { - } & \text { ción de discapacidad } \\
\text { - } & \text { Ligas deportivas } \\
\text { - } & \text { Institutos municipales de deportes y recreación } \\
\text { - } & \text { Centros de acondicionamiento físico }\end{array}$ \\
\hline
\end{tabular}




\begin{tabular}{|l|ll|}
\hline & $\bullet$ & Gimnasios \\
Administra- & $\bullet$ & Entes deportivos \\
ción y gestión & $\bullet$ & Ligas \\
deportiva & $\bullet$ & Clubes deportivos \\
& $\bullet$ & Escenarios deportivos \\
& $\bullet$ & Universidades \\
\hline
\end{tabular}

\section{Conclusiones}

La estructura administrativa de la práctica pedagógica en la Licenciatura en Educación Física de la Universidad de San Buenaventura, se acoge al esquema de la Facultad de Educación, en donde hay una coordinación al frente de las prácticas de todos los programas. Este esquema dificulta el desempeño mismo de la práctica no solo en términos de cantidad, sino también de saberes específicos, dado que la práctica en Educación Física por su carácter altamente procedimental y sus diversas áreas de desempeño, difiere de las prácticas en otros programas. En este sentido, es muy recomendable contar en la Licenciatura, con el apoyo de un docente con formación específica en el área, que apoye el proceso de la coordinación.

La práctica tiene presencia en todos los niveles de formación de la Licenciatura con cuatro modalidades que son también acogidas desde la facultad, como son docente, gestión pedagógica, social comunitaria e investigativa. En educación física se desarrollan prácticas en docencia, actividad física y salud, entrenamiento deportivo, administración deportiva e investigación; en ocasiones, la articulación o inclusión en las modalidades que presenta la facultad, se dificulta. Tener modalidades de acuerdo con las áreas de desempeño en la práctica, facilitaría el desarrollo de los procesos académicos.

Las modalidades son seleccionadas de manera voluntaria por los estudiantes sin un orden establecido; por lo tanto, un estudiante pudiera iniciar con una práctica en administración, luego de entrenamiento y finalmente de docencia, o bien, pudiera quedarse en una sola modalidad sin experimentar las otras áreas. Adicionalmente, puede presentarse que la práctica se desarrolle de manera desarticulada con los conteni- 
dos establecidos en el plan de estudios, dado que algunas asignaturas específicas se cursan posteriores a la práctica. Se propone que se establezca un orden coherente con el plan de estudios, de las modalidades de práctica en los niveles de formación.

En relación con los escenarios de práctica, prevalecen las instituciones educativas y se empieza a incursionar en otros escenarios como clubes deportivos y gimnasios; sin embargo, no se cuenta con una caracterización de los mismos, lo que contribuiría a una identificación de escenarios y modalidades para la asignación de la práctica.

Desde la percepción de directivos y docentes, la práctica presenta debilidades, como la asignación tardía de centros de práctica, la alta carga administrativa para ser asumida por una sola persona, el acompañamiento de un solo docente a grupos numerosos y de diversas modalidades, entre otras. En el caso de los estudiantes, casi el 50 \% presenta una percepción favorable; sin embargo, un porcentaje considerable de ellos $(21 \%)$, considera que el proceso no es adecuado.

Además de las recomendaciones descritas, la propuesta de adición de contenidos al plan de estudios, la caracterización de los centros y la inclusión de nuevos escenarios y modalidades de práctica, aportarían en perspectiva de mejoramiento continuo, a los procesos académico administrativos de la práctica en la Licenciatura en Educación Física y Deportes de la Universidad de San Buenaventura, además de mejorar la percepción que sobre ella tienen directivos, docentes y estudiantes.

\section{Bibliografía}

Álvarez de Zayas, C. M., González Agudelo, E. (2002). Lecciones de didáctica general. Bogotá: Magisterio.

Barragán, D. (2012). La práctica pedagógica: pensar más allá de las técnicas. En: Barragán D. y Urbina, J., Gamboa, A. Práctica Pedagógica. Discusiones teóricas. Cúcuta: U.F.P.S.

Gaitán, C. et al. (2005). Prácticas educativas y procesos de formación en la educación superior. Serie Estados del arte, Facultad de Educación. Bogotá: Facultad de Educación. Pontificia Universidad Javeriana. 
López , O. L., Cardona, J. M. y Muñoz, , D. (2006). Una propuesta integradora denominada "Proyecto Pedagógico". En Revista Itinerario Educativo. n. 47 de enero - junio de 2006. Bogotá: Universidad San Buenaventura.

Ministerio de Educación Nacional (2000). Lineamientos curriculares para el área de educación física, recreación y deportes. Bogotá: Magisterio.

Moreno, W. y Pulido, S. M. (2009). Universidad, currículo y Educación Física. Medellín: Funámbulos Editores.

Murillo Torrecilla, F. J. et al. (2006). Modelos innovadores en la formación inicial docente. Santiago de Chile: OREALC (Oficina Regional de Educación de la UNESCO para América Latina y el Caribe).

Posada, S. E. y Palacio, M. (2009). Documento de trabajo sobre el direccionamiento de la Facultad de Educación 2009-2011. Medellín: Universidad de San Buenaventura.

Universidad de San Buenaventura. Facultad de Educación. Proyecto pedagógico, una estrategia de aprendizaje de las prácticas. Disponible en http://proyectopedagogico.webnode.com.co/

Reglamento estudiantil (2001). Medellín: Universidad de San Buenaventura.

Ríos, B. E. (2011). Desde las prácticas pedagógicas: proyecto pedagógico una estrategia de aprendizaje que articula teoría-práctica-investigación. En La gestión educativa y el currículo en las prácticas contemporáneas. Medellín: Universidad de San Buenaventura.

Sacristán, G. (2010). Saberes e incertidumbres sobre el currículum. España: Morata.

Universidad de San Buenaventura, Rectoría General (2007). Proyecto Educativo Bonaventuriano. Bogotá: Editorial Bonaventuriana.

Universidad de San Buenaventura (2008). Cartilla interna: Sistema Integrado de Calidad. Medellín: Departamento de Publicaciones.

Universidad de San Buenaventura (2009). Reglamento de prácticas. Facultad de Educación. Medellín: Universidad de San Buenaventura. 
Universidad de San Buenaventura (2009). Informe de gestión y responsabilidad social 2009. Medellín: Universidad de San Buenaventura.

Universidad de San Buenaventura (2010). Estatuto Orgánico. Bogotá: Unidad de Publicaciones.

Universidad de San Buenaventura (2010). Portafolio de investigaciones. Bogotá: Unidad de Publicaciones.

Universidad de San Buenaventura (2010). Resolución de rectoría 030 del 27 de noviembre de 2010. Medellín: Universidad de San Buenaventura.

Universidad de San Buenaventura (2010). Facultad de Educación. Proyecto Educativo de Programa: Licenciatura en Educación Física y Deportes. Medellín: Universidad de San Buenaventura.

Universidad de San Buenaventura (2010) Resolución de Rectoría 004 del 1 de Febrero de 2010 por medio de la cual se modifica y actualiza el reglamento de práctica de la Universidad de San Buenaventura. Medellín: Universidad de San Buenaventura.

Universidad de San Buenaventura (s.f.). Documento interno de gestión del currículo DCPR.003.V1 